\title{
Life-Threatening Fournier's Gangrene Treated by Colostomy and Vacuum Assisted Closure
}

\author{
Jung Hwan Kim, Dong Lark Lee*, Hea Kyeong Shin, Gyu Yong Jung \\ Department of Plastic and Reconstructive Surgery, Dongguk University College of Medicine, Gyeongju, Korea
}

\begin{abstract}
Fournier's gangrene is a necrotizing fasciitis encountered in the scrotal and perineal region which progresses rapidly to a life-threatening state. Treatment options are dependent on the origin of the disease and lesion extent and depth. We report our experience of a case of Fournier's gangrene treated by colostomy and vacuum assisted closure (VAC). A 66-year-old man visited our emergency room with swelling and pain of his left buttock. After wide debridement of the perianal area and left buttock, VAC was applied for continuous drainage and to accelerate granulation. In addition, colostomy was performed to prevent fecal contamination. Four weeks after second debridement (with VAC and colostomy), we performed split thickness skin graft and local flap to successfully cover the wounds. We suggest VAC after early debridement and as a bolster dressing after skin grafting in managing Fournier's gangrene. We also recommend that if fecal contamination is suspected during the application of VAC, colostomy should be performed at early stage.
\end{abstract}

Keywords: Fournier gangrene, Colostomy, Vacuum

\section{Introduction}

Fournier's gangrene is a necrotizing fasciitis of the scrotum or perineum, which rapidly progresses to a life-threatening state. It has a high mortality rate, which ranges from $15-50 \%$, and thus, can be defined as an acute surgical emergency state [1]. Rapid fluid resuscitation, wide excision of devitalized tissues and parenteral administration of broad-spectrum antibiotics are mandatory for patients suffering from Fournier's gangrene [2]. The use of fecal or urinary diversion, vacuum assisted closure (VAC), and hyperbaric oxygen treatment have been advocated, but many factors including the origin, extent and depth of the disease should be considered before deciding on any specific course of treatment. However, the diversity of available options often causes confusion.

We hereby report our experience of a case of Fournier's gangrene treated by colostomy and VAC that had almost reached a life-threatening state and discuss the cautions associated with its treatment.

\section{Case}

A 66-year-old man was admitted to our general surgery department with swelling and pain of the left buttock. Initial physical examination revealed a heating sensation, redness and diffuse fluctuation of the left buttock and perineum, and severe swelling of the scrotum. Ultrasonography depicted a tubular hypoechoic lesion with subcutaneous swelling in the left perianal region (Fig. 1). A diagnosis of Fournier's gangrene

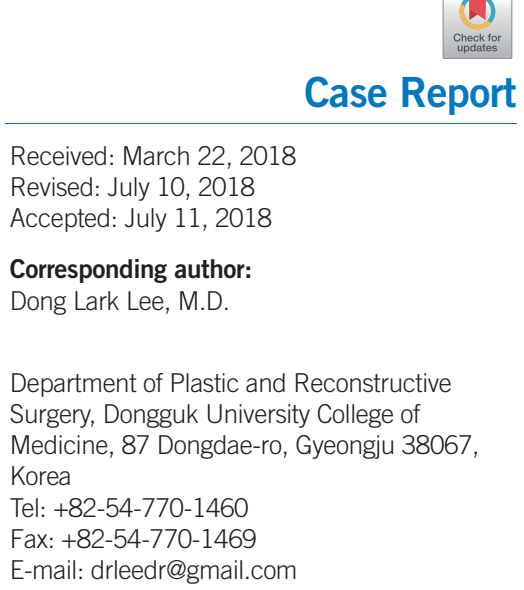

Case Report

Received: March 22, 2018

Revised: July 10, 2018

Accepted: July 11, 2018

\section{Corresponding author:}

Dong Lark Lee, M.D.

Department of Plastic and Reconstructive Surgery, Dongguk University College of Medicine, 87 Dongdae-ro, Gyeongju 38067, Korea

Tel: +82-54-770-1460

Fax: +82-54-770-1469

E-mail: drleedr@gmail.com

This is an Open Access article distributed under the terms of the Creative Commons Attribution Non-Commercial License (http://creativecommons.org/licenses/by-nc/4.0/) which permits unrestricted non-commercial use, distribution, and reproduction in any medium, provided the original work is properly cited.

(c) 2018 Korean Wound Management Society 


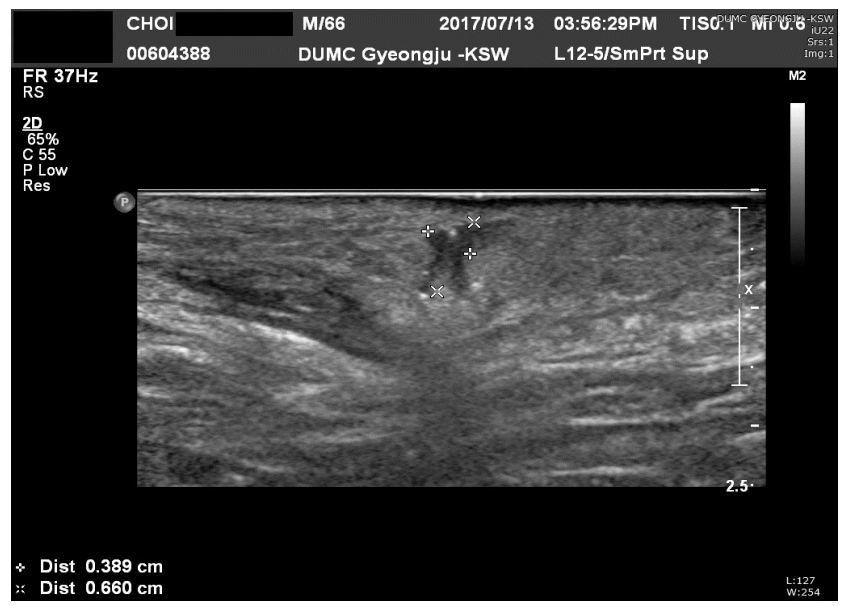

Fig. 1. Initial perianal ultrasonography revealed a tubular hypoechoic lesion with subcutaneous swelling in the left perianal area.

originating from a perianal fistula was reached. The patient was tachycardic and hypotensive, with a mean arterial pressure of 50-60 mmHg. Massive fluid loading with norepinephrine was administered through a central line and he was admitted to the Intensive Care Unit. Initial blood testing revealed the following: leucocytes $19,460 / \mu \mathrm{L}$; C-reactive protein (CRP) $31.70 \mathrm{mg} / \mathrm{dL}$; serum albumin, $2.5 \mathrm{~g} / \mathrm{dL}$; and Hemoglobin A1c (HbA1c), 9.7\%. Emergency debridement was performed at the general surgery department, and the patient was transferred to the plastic and reconstructive surgery department for wound management and reconstruction of the perineal area. Escherichia coli and Streptococcus agalactiae were detected from the wound culture results at the initial debridement. Aminoglycosides (amikacin) and 3rd generation cephalosporins (cefotaxime) were administered according to the results of antibiotic sensitivity. Despite initial debridement and antibiotics administration, the left buttock was still swollen with redness and fluctuation. Scrotal skin and fascia had been almost sacrificed during initial debridement, and remaining necrotic tissues also required additional debridement (Fig. 2).

Wide and aggressive debridement of the perianal area was performed during a second surgical procedure. A long incision was made from scrotum to the left buttock to enable complete resection of the perianal fistula. After debridement, we immediately applied vacuum assisted closure (VAC) for continuous drainage and to accelerate granulation. VAC dressings were exchanged twice every week and wound culture was performed at every exchange. Follow-up cultures taken 10 days after starting antibiotics were negative for Escherichia coli and

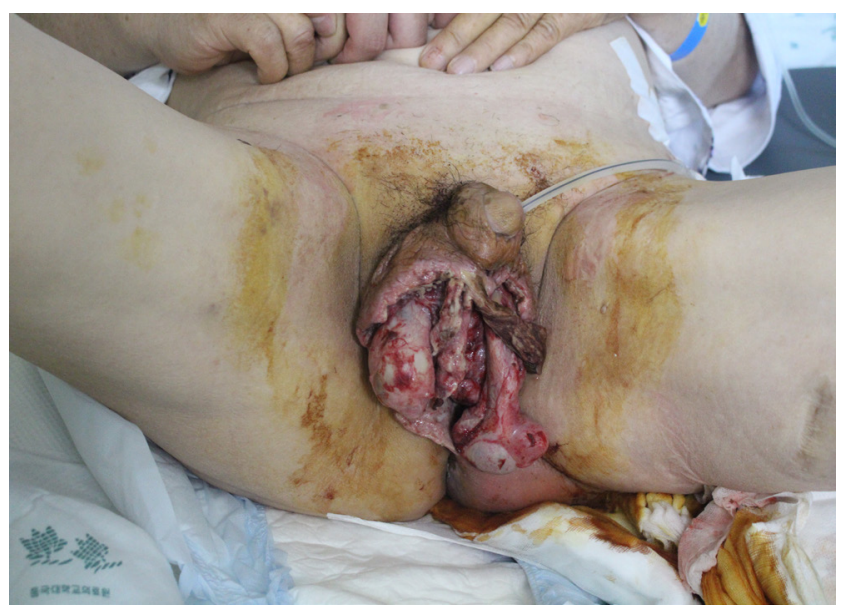

Fig. 2. Scrotal skin and fascia were almost sacrificed during initial debridement, and remaining necrotic tissues needed additional debridement.

Streptococcus agalactiae; Enterococcus faecium was detected in their stead. Cefotaxime was continuously administered according to sensitivity results. As the lesion extended from the perineum to the scrotal area, a very convoluted and moistureprone area, initially VAC application proved to be very difficult. In addition, it was impossible to shape the VAC sponge without affecting normal skin and testis parenchyma while maintaining an airtight seal. Therefore, rather than designing the sponge in a complicated manner, we made a large rectangular shape to simply cover the wound area (Fig. 3). Because of concerns that VAC would be contaminated by feces, we ordered total parenteral nutrition (TPN). However, even though the anus was not included in the VAC dressing the wound was continuously contaminated by feces leaking into the VAC sponge. Three days after the second debridement, VAC was removed due to persistent fecal contamination and colostomy was performed two days later. Since fecal anal discharge may continue for several days after colostomy, we maintained povidone-iodine soaking dressings for one more week. After confirming the cessation of fecal discharge, VAC was re-applied and retained negative pressure remarkably well with minimal fecal contamination, which proved significantly advantageous in wound management.

4 weeks after the second surgical debridement (with VAC and colostomy), the infection of the scrotum, inguinal area, and left buttock was under control (Fig. 4). Follow-up blood tests displayed improved leukocyte $(5,970 / \mu \mathrm{L})$ and CRP $(1.42$ $\mathrm{mg} / \mathrm{dL}$ ) levels, and the patient himself was in good general condition with normal vital signs. Although culture results 


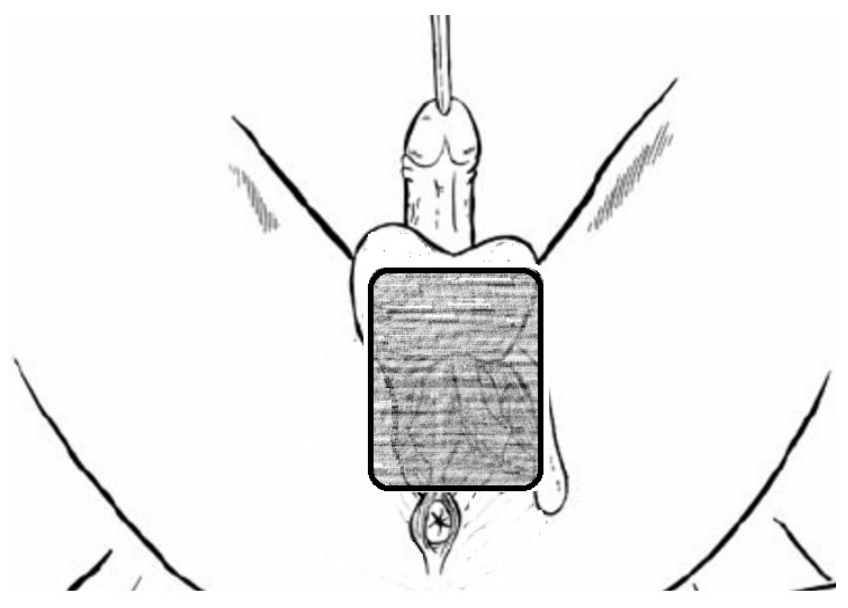

Fig. 3. We designed the shape of the VAC sponge in a large rectangular shape to apply VAC stably.

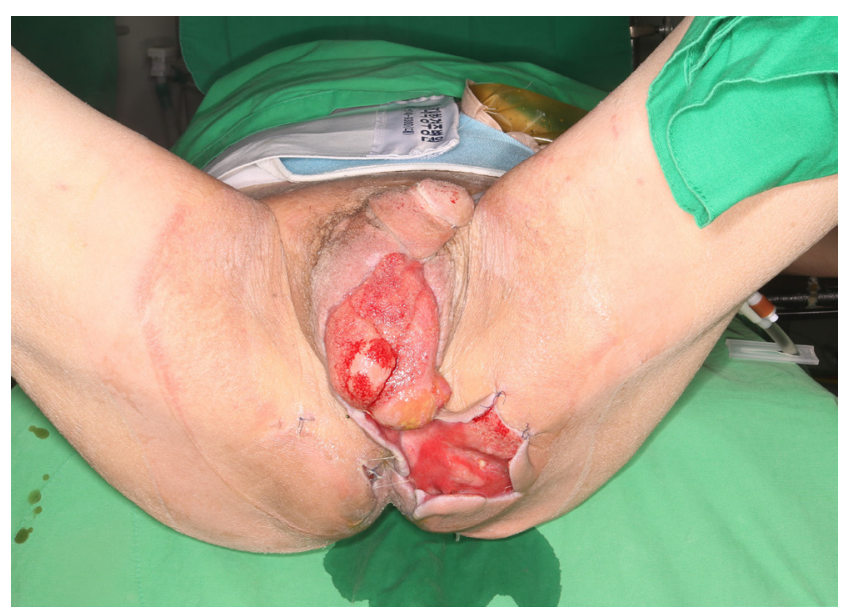

Fig. 4. Three weeks after second debridement (with VAC and colostomy), infection of the scrotum, inguinal area, and left buttock was controlled.

continued to be positive for Enterococcus faecium, from a clinical standpoint, the infection was judged to be under control and a decision was made for surgical reconstruction. Reconstruction was performed using multiple methods. The scrotum was covered by a split-thickness skin graft, and the perineal area and buttock were reconstructed with a bilateral advancement flap (Fig. 5). VAC was applied as a bolster dressing over the split-thickness skin graft for 5 days.

The skin graft had completely taken to the scrotum at 14 days after reconstructive surgery (POD14), but dehiscence occurred in the perineum and buttock surgical wound, and thus, minimal debridement was performed and wound edges were approximated using a local advancement flap to cover the de-

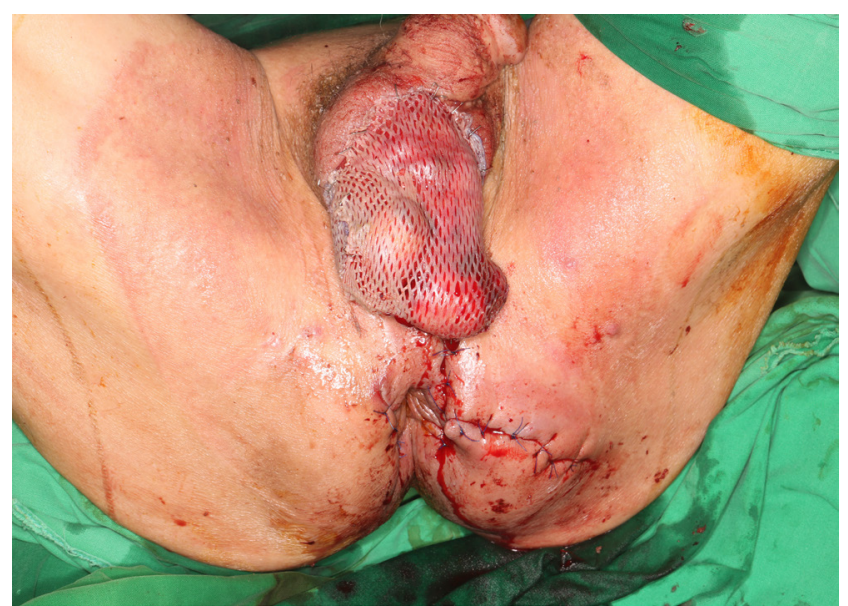

Fig. 5. The scrotum was covered by a split-thickness skin graft, and the perineal area and buttock were covered by a bilateral advancement flap.

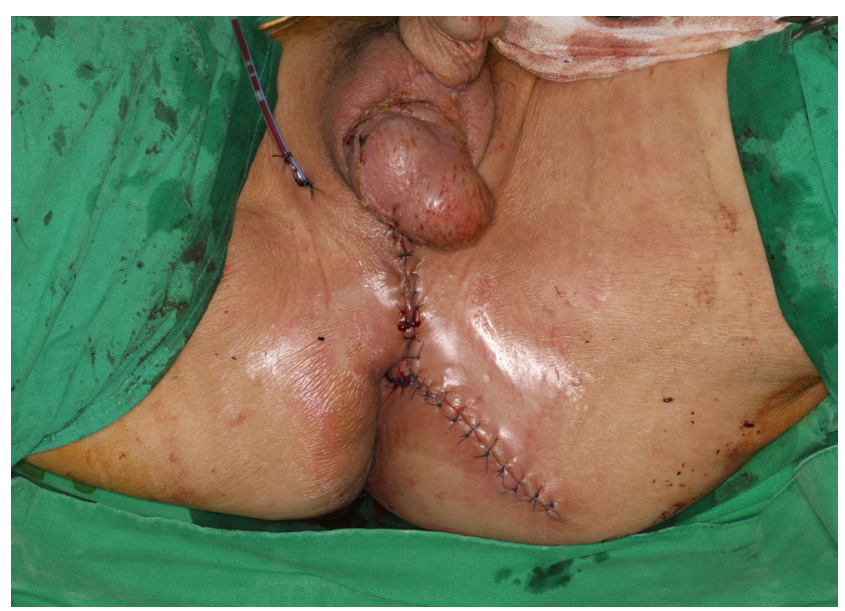

Fig. 6. Dehiscence occurred in surgical wounds of perineum and buttock at 14 days after reconstructive surgery, and thus, minimal debridement was performed. Wound edges were approximated using a local advancement flap to cover the defect.

fect (Fig. 6). On POD 30, the surgical wound had completely healed and the colostomy was closed. On POD 60, there were no remarkable complications and his anal function was intact (Fig. 7).

\section{Discussion}

Fournier's gangrene was first described by Alfred Fournier in 1883 as an infective necrotizing fasciitis of the genital and perianal region [3]. Inflammation from bacterial infection promotes bacterial spread through the fascial layer and progresses to gangrene of subcutaneous tissues and skin [4]. The clinical manifestations of Fournier's gangrene include perineal 


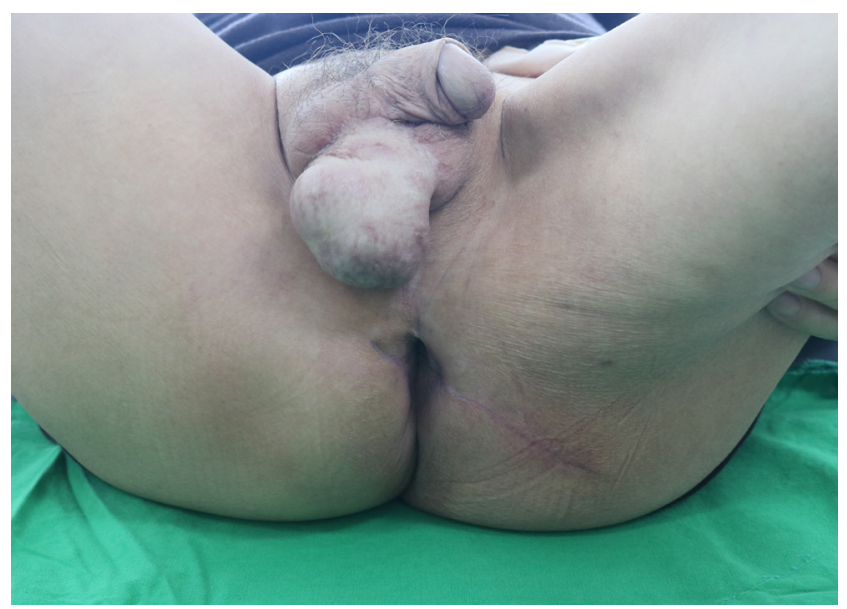

Fig. 7. At 60 days after reconstructive surgery, there was no remarkable complication and anal function was intact.

pain, crepitation, bulla, and purulent discharge and systemic symptoms of fever, septic shock, and/or multiorgan failure. The diagnosis of Fournier's gangrene is primarily based on clinical findings of fluctuance, crepitus, localized tenderness, and wounds of the genitalia and perineum [4].

General treatment principles are rapid, aggressive surgical debridement, hemodynamic support with urgent fluid resuscitation, and broad-spectrum parental antibiotics. In our case, according to these principles, we monitored vital signs and maintained blood pressure with massive fluid resuscitation. Debridement was performed, and simultaneously, infection and inflammation were controlled with parental antibiotics using wound culture results for guidance.

However, the dressing of wounds after the surgical treatment of Fournier's gangrene has not been clearly established, which makes decisions difficult. According to Chennamsetty et al. [4], topical therapy, vacuum assisted closure, and hyperbaric oxygen therapy were most commonly used, and of these, we chose VAC as the dressing tool.

The using of VAC for dressing has several advantages. Ozkan et al. stated that VAC increases blood supply and accelerates the formation of granulation tissue by removing bacterial contamination [5]. Since the VAC exchange cycle is longer than that of other dressings, we dressed wounds twice weekly, which means VAC significantly reduced pain associated with dressing. In addition, dressing durability is considerably better than those of other dressings. Conventional dressings on the groin or perineum are prone to dislodge when wet and easily get intertwined or loosened, whereas the vacuum of VAC secures the dressing during positional changes and while walking.
Furthermore, applying VAC to skin grafts can significantly increase skin graft take compared to conventional methods because it creates a closer contact between the graft and the wound bed, and prevents the accumulation of blood or exudate beneath the graft through active evacuation [6,7]. We also applied VAC after split-thickness skin grafting on these bases, to prevent seroma or hematoma accumulation which has a higher risk of occurring on curved surfaces, and to ensure graft fixation with the wound bed.

One problem we encountered while applying VAC was persistent stool contamination. TPN was initially used in an attempt to prevent VAC fecal contamination but proved ineffective. As TPN also had a risk of reducing immunological competence we performed a colostomy. Although opinions are divided regarding the need for colostomy, Chennamsetty et al. recommended that anal sphincter involvement, fecal incontinence and continued fecal contamination of wound margins be viewed as indications for colostomy [4]. Because Fournier's gangrene is vulnerable to fecal contamination, we recommend performing colostomy during initial debridement if there is any doubt regarding possible fecal contamination [8].

Fournier's gangrene has a high mortality rate despite aggressive multidisciplinary treatment. We suggest that VAC after early debridement aids treatment and that VAC be considered after split thickness skin graft reconstruction. Finally, we recommend that if fecal contamination is suspected during the application of VAC, colostomy should be performed early.

\section{References}

1. Eke N. Fournier's gangrene: a review of 1726 cases. $\mathrm{Br} \mathrm{J}$ Surg 2000;87:718-28.

2. Yanar $\mathrm{H}$, Taviloglu $\mathrm{K}$, Ertekin $\mathrm{C}$, et al. Fournier's gangrene: risk factors and strategies for management. World J Surg 2006;30:1750-4.

3. Thwaini A, Khan A, Malik A, et al. Fournier's gangrene and its emergency management. Postgrad Med J 2006;82:5169.

4. Chennamsetty A, Khourdaji I, Burks F, et al. Contemporary diagnosis and management of Fournier's gangrene. Ther Adv Urol 2015;7:203-15.

5. Ozkan O, Koksal N, Altinli E, et al. Fournier's gangrene current approaches Int Wound J 2016;13:713-6.

6. Orhan E, Şenen D. Using negative pressure therapy for improving skin graft taking on genital area defects following Fournier gangrene. Turk J Urol 2017;43:366-70. 
Kim JH et al.

Fournier's gangrene

7. Moisidis E, Heath T, Boorer C, et al. A prospective, blinded, randomized, controlled clinical trial of topical negative pressure use in skin grafting. Plast Reconstr Surg 2004;114: 917-22.
8. Akcan A, Sözüer E, Akyildiz $H$, et al. Necessity of preventive colostomy for Fournier's gangrene of the anorectal region. Ulus Travma Acil Cerrahi Derg 2009;15:342-6. 